\title{
Calculated oxygen $1 s$ core-level photoemission spectra from cuprate superconductors
}

\author{
A CHAINANI and D D SARMA \\ Solid State and Structural Chemistry Unit, Indian Institute of Science, Bangalore 560012, \\ India \\ MS received 14 January 1991; revised 25 April 1991

\begin{abstract}
The observed O $1 \mathrm{~s}$ X-ray photoemission spectra of the cuprate superconductors often exhibit a satellite at a higher binding energy to the main peak. The origin of this satellite is not fully understood. We have done model cluster calculations to investigate the origin of this satellite using the configuration interaction approach. The calculated spectra for the divalent and superconducting cuprates essentially exhibit a single peak. On distorting the cluster in-plane, the peak shifts to higher binding energies. This substantiates a deterioration of the surface leading to the observed satellite structure in the $\mathrm{O} 1 \mathrm{~s}$ core-level photoemission spectra of the cuprate superconductors.
\end{abstract}

Keywords. X-ray photoemission spectra; oxygen $1 s$; cuprate superconductors; core-level calculation.

PACS Nos 74.70; 79.60; 71.28

\section{Introduction}

The oxygen 1s photoemission spectra from metal oxides have rarely shown prominent features besides the main peak. This is in contrast to the metal core levels which show additional features in many cases e.g. the transition metal $2 p$ spectra from formally divalent $\mathrm{Ni}^{2+}$ compounds (Zaanen et al 1986) and $\mathrm{Cu}^{2+}$ compounds (van der Laan et al 1981), rare-earth $3 d$ spectra from the early lanthanide compounds (Gunnarsson and Schönhammer 1983a, b, 1985) and the $4 f$ spectra of the actinides (Gunnarsson et al 1985; Sarma et al 1986; Sarma et al 1987a). But recently, numerous investigations have been reported on the ligand level spectra of the copper-based high $T_{c}$ superconductors (Sarma et al 1987b; Weaver et al 1988; Takahashi et al 1988; Arko et al 1989; Hill et al 1987), as it is generally believed that the oxygen derived states play an important role in the mechanism of superconductivity in these compounds. Many of these studies indicate an extra feature in the $\mathrm{O} 1 \mathrm{~s}$ spectra at a higher binding energy besides the main peak. However the interpretation of this extra feature as an intrinsic signal has been questioned. The main reason for this is that the intensity of this extra feature (the satellite) has been found to be strongly dependent on the sample surface preparation.

Early reports ascribed the cause of this feature to be contaminant-related (Sarma et al 1987b). Takahashi et al have reported $\mathrm{O} 1 \mathrm{~s}$ spectra for single crystalline $\mathrm{La}_{2-x} \mathrm{Sr}_{x} \mathrm{CuO}_{4}$ which shows a single peak structure in both the undoped and Sr-doped crystals for a freshly cleaved surface at low temperature, but transforms into 
a double peak structure at room temperature. Also, studies (Weaver et al 1988) on single crystalline $\mathrm{YBa}_{2} \mathrm{Cu}_{3} \mathrm{O}_{7}$ have shown the $531 \mathrm{eV}$ peak being related to surface degradation. However the nature of the surface degradation is not very clear. Many authors have attributed this feature to surface impurities like hydroxyls and carbonates, while others have suggested the formation of defects as responsible. Yet some authors have interpreted the extra feature as intrinsic.

\section{Calculation}

To understand the nature of this extra feature, we performed calculations based on the single impurity Anderson Hamiltonian for a cluster to obtain the O 1 s core level photoemission spectra. The cluster (figure la) consists of one copper atom surrounded by four oxygen atoms in a square planar arrangement having the $D_{4 h}$ symmetry. This cluster is representative of the copper oxygen sheets in the copper based superconductors. We have also calculated the photoemission spectra for the clusters

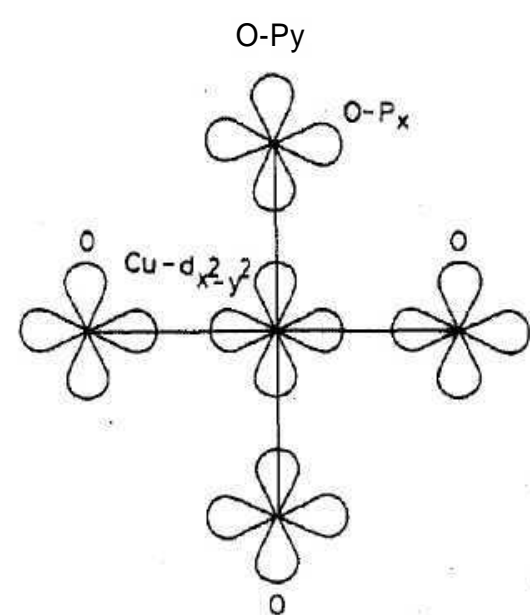

(a)

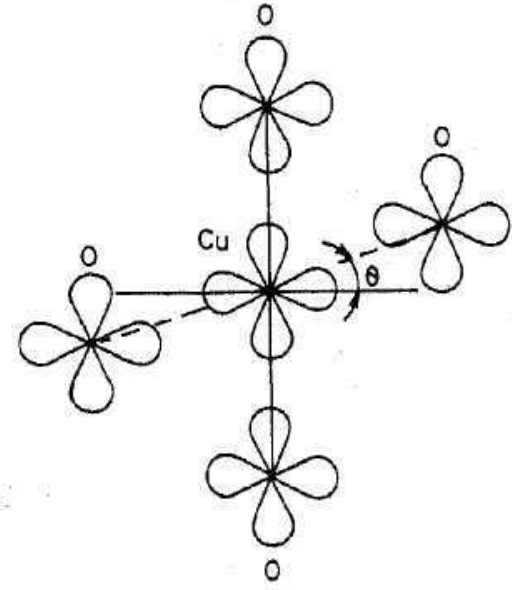

(c)

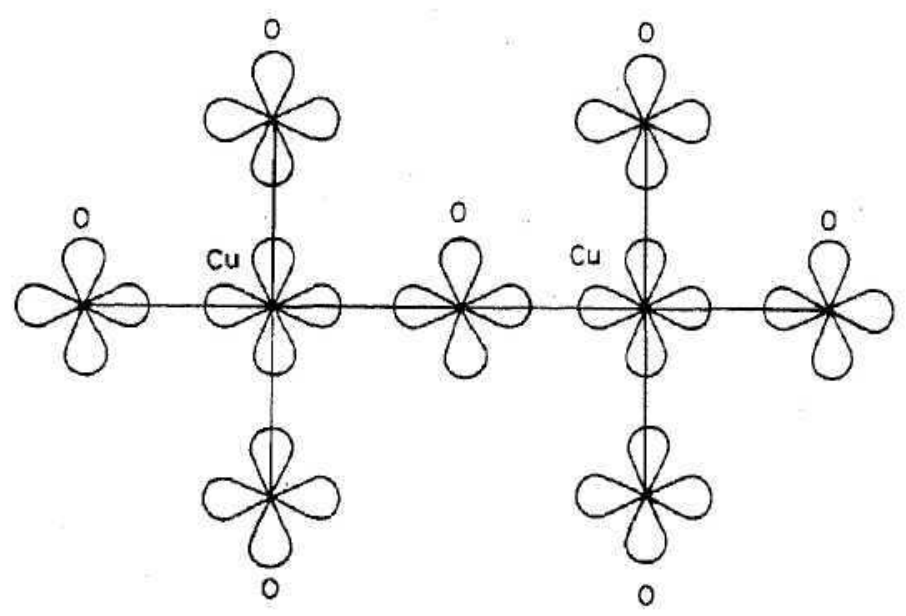

(b)

Figure 1. (a) The $\mathrm{CuO}_{4}$ cluster used for the $\mathrm{O} 1 \mathrm{~s}$ core-level calculation. (b) The $\mathrm{Cu}_{2} \mathrm{O}_{7}$ cluster for checking size-eflects. (c) The distorted $\mathrm{CuO}_{4}$ cluster showing the angle of distortion. 
shown in figures $1 \mathrm{~b}$ and $1 \mathrm{c}$; the significance of these clusters will be discussed later. We consider a $d_{x^{2}-y^{2}}$ orbital at the copper site and $p_{x}$ and $p_{y}$ orbitals at each oxygen site, with a single hole in the $\mathrm{CuO}_{4}$ cluster corresponding to the formally divalent configuration, as in $\mathrm{La}_{2} \mathrm{CuO}_{4}$. A state with two holes in the cluster, in this sense, corresponds to the $d^{8}$ configuration, or the trivalent state. In presence of hybridization, we then have, in the hole picture, basis states of the type $\left|d^{0} p^{1}\right\rangle$ and $\left|d^{1} p^{0}\right\rangle$ for the divalent case and $\left|d^{2} p^{0}\right\rangle,\left|d^{1} p^{1}\right\rangle$ and $\left|d^{0} p^{2}\right\rangle$ for that of the trivalent.

The Hamiltonian describing the cluster consists of the bare energies ( $E_{p}$ and $E_{d}$ with $\left.\mathrm{A}=E_{p}-E_{d}\right)$, the hopping matrix elements $\left(V_{p d}, V_{p p \sigma}\right.$ and $\left.V_{p p \pi}\right)$ between copper and oxygen as well as between near-neighbour oxygens, intrasite Coulomb energies $\left(U_{d d}\right.$ and $\left.U_{p p}=U_{p_{x} p_{x}}=U_{p_{x} p_{y}}=U_{p_{y} p_{y}}\right)$, an intersite Coulomb energy $\left(U_{p d}\right)$ and an onsite core hole $(\mathrm{O} 1 s)$ - valence hole $(\mathrm{O} 2 p)$ Coulomb energy $\left(U_{p c}\right)$.Using configuration interaction for the initial and final states of the system and assuming the sudden approximation for the photoemission process (Gunnarsson and Schönhammer 1983a,b; 1985), we obtain the calculated O $1 s$ photoemission spectra for the said clusters. The discrete energies obtained for the possible states are broadened by an instrumental resolution width $(1.0 \mathrm{eV})$ and an energy dependent lifetime broadening. The strength, $U_{p c}$, between the core hole in the $\mathrm{O} 1 \mathrm{~s}$ level and a hole in the $2 p$ level, is taken to be $10 \mathrm{eV}$ in all cases. Similar calculations have been performed earlier and the details can be obtained in Sarma (1988) and Sarma and Taraphder (1989).

\section{Results}

We first consider the cases of the divalent oxides (e.g. $\mathrm{CuO}, \mathrm{La}_{2} \mathrm{CuO}_{4}$ ). We assume $V_{p p \sigma}=-1.0 \mathrm{eV}$ and $V_{p p \pi}=0.0 \mathrm{eV}$, leading to a total oxygen $2 p$ related bandwidth of $4.0 \mathrm{eV}$ (Sarma and Taraphder 1989) and $V_{p d}$ is taken to be $-1.15 \mathrm{eV}$. A was chosen to be $-3 \cdot 0 \mathrm{eV}$ so as to give a total $d$-electron count of $\sim 9 \cdot 3$ (which conforms to the divalent case of $\mathrm{CuO}$ ). For these parameters, the calculated spectrum is as shown in figure 2a. It consists of a single peak which we have fixed at $529 \mathrm{eV}$ binding energy. To check for cluster size-effects on the results, we did the same calculation for a cluster of two copper and seven oxygen atoms arranged in plane having the $C_{2 v}$ symmetry (figure $1 \mathrm{~b}$ ).

For the cluster shown in figure 1(b), the calculation of the $\mathrm{O}$ 1s spectra was done for the middle oxygen atom which has a different environment as compared to an oxygen atom in the cluster shown in figure 1(a). This change in the environment would result in a higher binding energy of the $\mathrm{O} 1 \mathrm{~s}$ level. For a $\mathrm{A}$ of $-2.7 \mathrm{eV}$, $V_{p d}=-1.15 \mathrm{eV}, V_{p p \sigma}=-1.0 \mathrm{eV}, U_{d d}=14.0 \mathrm{eV}, U_{p p}=6.0 \mathrm{eV}$ and $U_{p d}=4.0 \mathrm{eV}$ we once again obtain an $n_{\mathbf{d}}$ value of $\sim \mathbf{9 . 3}$ and a single peak as shown in figure 2(b). Without any change in the spectral shape, the O $1 \mathrm{~s}$ core-level for the cluster shown in figure 1(b) shifts to higher binding energy by $0.5 \mathrm{eV}$ as compared to the cluster shown in figure 1(a). Thus, the finite size of the cluster does not pose a serious limitation since there is no change in the shape of the calculated spectra. Within the divalent case, if the magnitude of $\mathrm{A}$ is decreased so as to reproduce a higher $\boldsymbol{n}_{\boldsymbol{d}}$ value (e.g. as in $\mathrm{La}_{2} \mathrm{CuO}_{4}$, Sarma 1988), the calculated spectrum continues to exhibit a single peak (figure 2c). For the superconducting case, using parameter values as estimated (Sarma and Taraphder 1988) (namely, $\Delta=-1.0 \mathrm{eV}, V_{p d}=-1.15 \mathrm{eV}$, $V_{p p \sigma}=-1.0 \mathrm{eV}, V_{p p \pi}=0.0 \mathrm{eV}, U_{d d}=12.0 \mathrm{eV}, U_{p p}=6.0 \mathrm{eV}$ and $\left.U_{p d}=3.0 \mathrm{eV}\right)$ and the 


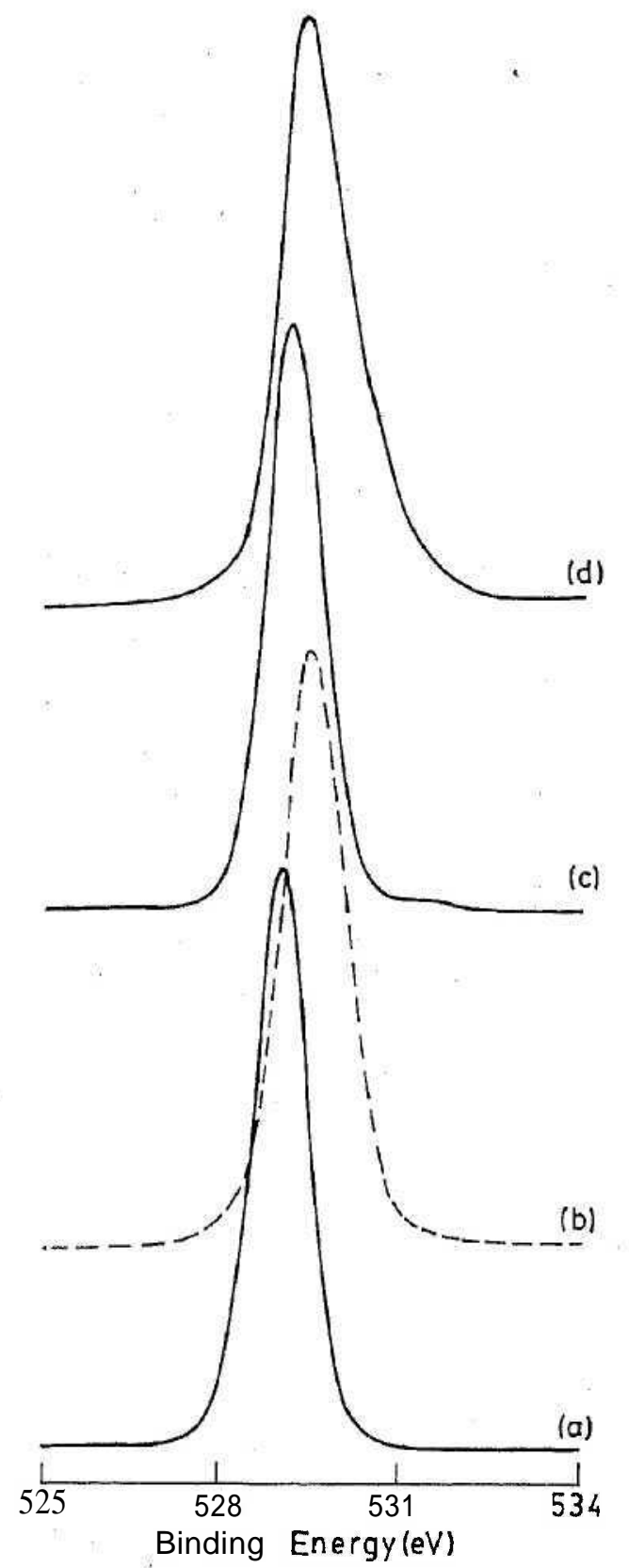

Figure 2. The calculated $\mathrm{O} 1 s$ spectra for (a) $V_{p d}=-1.15 \mathrm{eV}, V_{p l \sigma}=-1.0 \mathrm{eV}, \Delta=-3.0 \mathrm{eV}$ $\left(\mathrm{CuO}_{4}\right.$ duster for $\left.\mathrm{CuO}\right),(\mathrm{b}) V_{p d}=-1.15 \mathrm{eV}, V_{p p q}=-1.0 \mathrm{eV}, \mathrm{A}=-2.7 \mathrm{eV}, U_{d d}=14.0 \mathrm{eV}$, $U_{p p}=6.0 \mathrm{eV}, U_{p d}=4.0 \mathrm{eV}\left(\mathrm{Cu}_{2} \mathrm{O}_{7}\right.$ cluster for $\left.\mathrm{CuO}\right)$, (c) parameters same as in (a) but with $\Delta=-1.0 \mathrm{eV}\left(\mathrm{CuO}_{4}\right.$ cluster for $\left.\mathrm{La}_{2} \mathrm{CuO}_{4}\right)$ and (d) $V_{p d}=-1.15 \mathrm{eV}, V_{p p o}=-1.0 \mathrm{eV}, \mathrm{A}=$ $-1.0 \mathrm{eV}, U_{d d}=12.0 \mathrm{eV}, U_{p p}=6.0 \mathrm{eV}$ and $U_{p t}=3.0 \mathrm{eV}$ (for the superconducting cuprates).

$D_{4 h}$ cluster with two holes, we obtain the spectrum shown in figure $2 \mathrm{~d}$. The spectrum consists of a main peak and a weaker signal $1.0 \mathrm{eV}$ above the main peak (i.e. at about $530 \mathrm{eV}$ binding energy). The combination of these two peaks shows up as a single asymmetric peak in the final spectrum (figure $2 \mathrm{~d}$ ). The origin of the second peak that leads to the asymmetric feature in this case has been discussed in a previous publication (Sarma and Chainani 1990). Thus, even for the higher valency state, we essentially obtain a single (though asymmetric) peak spectrum, which does not explain the observed second peak at about $532 \mathrm{eV}$ binding energy in the experimental $\mathrm{O} 1 \mathrm{~s}$ 


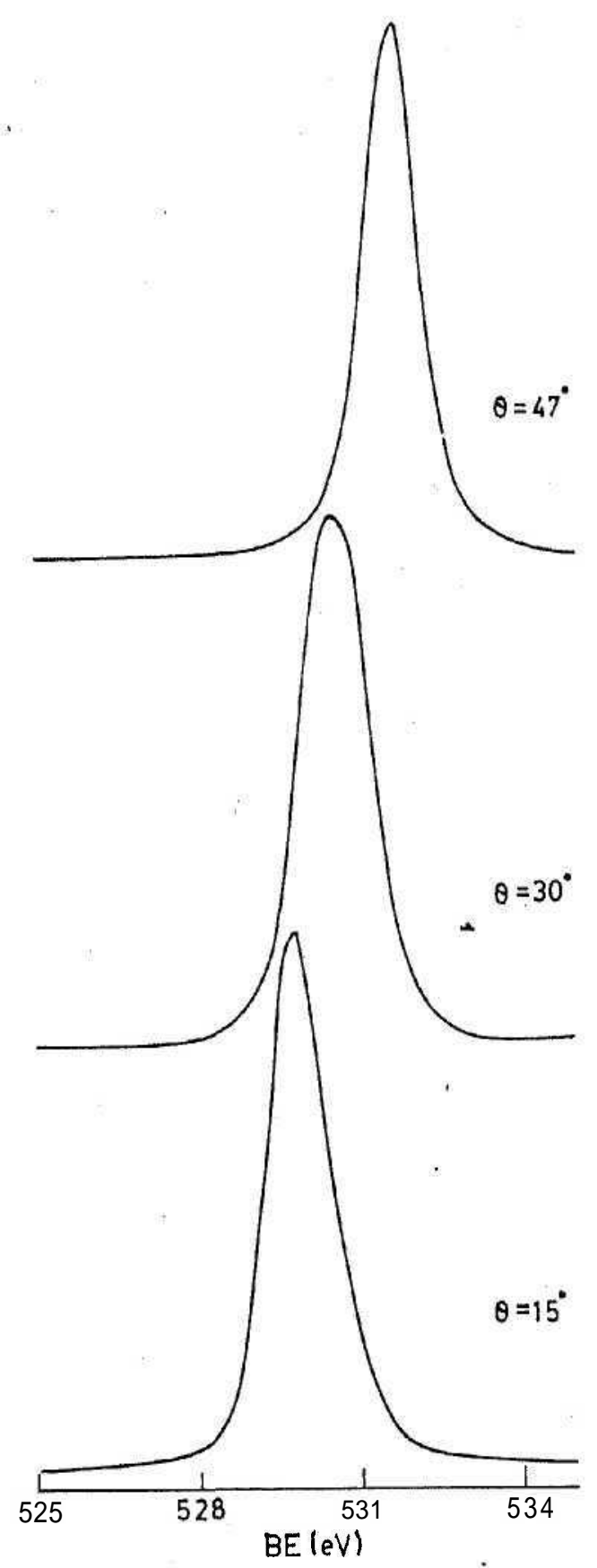

Figure 3. The calculated spectra for the distorted $\mathrm{CuO}_{4}$ for 0 values of $15^{\circ}, 30^{\circ}$ and $47^{\circ}$ showing the peak moving to higher binding energies.

spectrum. It is to be noted that such asymmetry indicating a higher peak at about $530 \mathrm{eV}$ has been reported from experiments of several single crystalline samples (Weaver et al 1988). The distinct higher energy peak that is often seen in the $\mathrm{O} 1 \mathrm{~s}$ spectra of the superconductors is, however, nearly at $532 \mathrm{eV}$. It is interesting that this energy position matches with that from the peroxo-species (Joyner and Roberts 1979 and Campbell 1986). Since the oxygen atoms in a peroxide form shorter bonds, we distorted the $\mathrm{CuO}_{4}$ cluster in plane so that the oxygen atoms move towards each other as shown in figure 1c. We have used the parameters, $\mathrm{A}=-1 \cdot 0 \mathrm{eV}, V_{p d}=-1.15 \mathrm{eV}$, $V_{p p \sigma}=-1.0 \mathrm{eV}, U_{d d}=12.0 \mathrm{eV}, U_{p p}=6.0 \mathrm{eV}$ and $U_{p d}=3.0 \mathrm{eV}$ and the hybridization 
between nearest neighbour oxygen atoms is taken to vary as $r^{-3}$ where $r$ is the distance between the oxygen atoms (Andersen et al 1978). The calculated spectra for various values of 9 are shown in figure 3. The spectra show a single peak which moves to higher binding energy for increasing 0 . For a distance of $1.4 \hat{A}$ between two oxygen atoms (typical of a peroxo bond), the peak appears at $531 \cdot 7 \mathrm{eV}$, in good agreement with the often observed second peak in the $\mathrm{O} 1 \mathrm{~s}$ spectra of the superconductors. Here we note that while the undistorted cluster (figure 1(a)) did not require $d_{x y}$ interactions to be included, the full treatment of the distorted cluster (figure 1(c)) should include $d_{x y}$ interactions. Though neglecting it is a limitation of the calculation, we expect it to play a less significant role. In fact, we find that it is the oxygen-oxygen interaction that is responsible for the increase in the binding energy observed in figure 3.

Thus, it appears that the second feature in the O 1 s core-level spectra may partly be due to the formation at the surface of $\mathrm{O}_{2}^{2-}$ or similar valent species. This interpretation is not unreasonable, since the absence of the next layer (and the consequent strong modification of the potential at the surface) will allow a substantial relaxation of the $\mathrm{Cu}-\mathrm{O}$ arrangement away from the bulk square-planar symmetry. While the surface modification can, in principle, be calculated from first principle calculations, we consider it to be beyond the scope of this work. It is also to be admitted that the other common impurities (e.g. hydroxyl and carbonates) at the surface will also contribute extra features, thus making an experimental estimation of the contribution of the surface distortion to the second peak difficult.

\section{References}

Andersen O K, Klose W and Nohl H 1978 Phys. Rev. B17 1209

Arko A J et al 1989 Phys. Rev. B40 2268

Campbell C T 1986 Surf. Sci. 173 L641

Gunnarsson O and Schonhammer K 1983a Phys. Rev. Lett. 50604

Gunnarsson O and Schonhammer K 1983b Phys. Rev. B28 4315

Gunnarsson 0 and Schonhammer K 1985 Phys. Rev. 314815

Gunnarsson O, Schonhammer K, Sarma D D, Hillebrecht F U and Campagna M 1985 Phys. Rev. B32 5499 Hill D M, Meyer III H M, Weaver J H, Flandermeyer B and Capone II D W 1987 Phys. Rev. B36 3979 Joyner R W and Roberts M W 1979 Chem. Phys. Lett. 60459

Sarma D D, Hillebrecht F U, Gunnarsson O and Schonhammer K 1986 Z. Phys. B63 305

Sarma D D, Hillebrecht F U and Brooks M S S 1987a J. Magn. Mater. 63-64 509

Sarma D D, Sreedhar K, Ganguly P and Rao C N R 1987 Phys. Rev. B36 2371

Sarma D D 1988 Phys. Rev. B37 7948

Sarma D D and Taraphder A 1989 Phys. Rev. B39 1570

Sarma D D and Chainani A 1990 Phys. Rev. B41 6688

Takahashi T, Maeda F, Katayama-Yosida H, Okabe Y, Suzuki Y, Fujimori A, Hosoya S, Shamoto S and Sato S 1988 Phys. Rev. B37 9788

Van der Laan C, Westra C, Haas C and Sawatzky G A 1981 Phys. Rev. B23 4369

Weaver J H, Meyer III H M, Wagner T J, Hill D M, Gao Y, Peterson D, Fisk Z and Arko A J 1988 Phys. Rev. B38 4668

Zaanen J, Westra C and Sawatzky G A 1986 Phys. Rev. B33 8060 